Revista Thema

v.20 Especial 2021

p.239-251

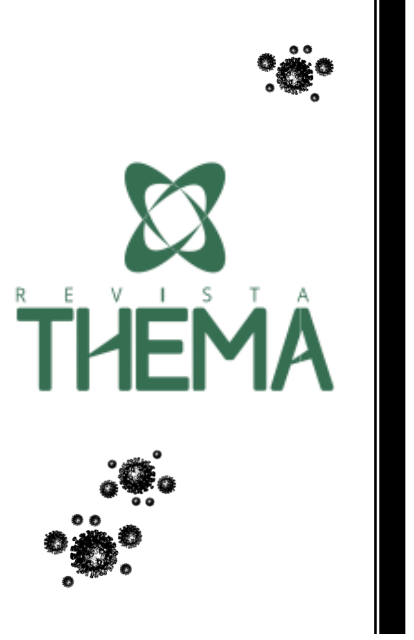

DOI: http://dx.doi.org/10.15536/thema.V20.Especial.2021.239-251.1908

ISSN: 2177-2894 (online)
CIÊNCIAS DA SAÚDE

\section{Burnout em docentes do ensino superior no período da pandemia da COVID-19:} reflexões com estudantes de enfermagem

\author{
Burnout in higher education teachers in the COVID-19
}

pandemic period: reflections with nursing students

Beatriz Maria dos Santos Santiago Ribeiro', Lucas Rossato², Fabio Scorsolini-Comin ${ }^{3}$

\title{
RESUMO
}

Na docência no ensino superior, o burnout constitui uma problemática cada vez mais emergente. No contexto da pandemia da COVID-19, os contextos laborais do ensino superior passaram por modificações significativas, repercutindo nas condições para o trabalho docente e nos processos de ensino-aprendizagem. O objetivo deste relato é descrever uma experiência profissional vivenciada durante uma discussão virtual sobre a síndrome de burnout com estudantes de um curso de graduação em Enfermagem, produzido a partir da disciplina de Saúde Ocupacional. Os discentes apontaram que a formação profissional em saúde ocupacional é determinante na atenção aos processos de promoção de saúde nesse contexto. A figura do docente de nível superior foi destacada como uma das mais afetadas pela pandemia. Estratégias de prevenção e de promoção de saúde foram endereçadas. O olhar amadurecido para todas as ressonâncias no mundo do trabalho e na saúde dos profissionais de educação deve contribuir para que os estudantes de Enfermagem construam posicionamentos críticos para a condução de discussões cada vez mais emergentes no cenário pós-pandemia.

Palavras-chave: Esgotamento profissional; distanciamento social; COVID-19.

\section{ABSTRACT}

In teaching in higher education, burnout is an increasingly emerging problem. In the context of the COVID-19 pandemic, the higher education work contexts underwent significant changes, affecting the conditions for teaching work and the teaching-learning processes. The objective of this report is to describe a professional experience lived during a virtual discussion about the burnout syndrome with students of a Nursing course, produced from the Occupational Health discipline. The students pointed out that professional training in occupational health is decisive in the attention to health promotion processes in this context. The figure of the higher

\footnotetext{
${ }^{1}$ Doutoranda em Enfermagem, Escola de Enfermagem de Ribeirão Preto - EERP, Universidade de São Paulo - USP, Ribeirão Preto/SP - Brasil. E-mail: beatrizsantiago1994@hotmail.com

2 Doutorando em Enfermagem, Escola de Enfermagem de Ribeirão Preto - EERP, Universidade de São Paulo - USP, Ribeirão Preto/SP - Brasil. E-mail: rossatousp@usp.br

${ }^{3}$ Professor Associado, Escola de Enfermagem de Ribeirão Preto - EERP, Universidade de São Paulo - USP, Ribeirão Preto/SP - Brasil. E-mail: fabio.scorsolini@usp.br
} 
education teacher was highlighted as one of the most affected by the pandemic. Prevention and health promotion strategies were addressed. The mature look at all the resonances in the world of work and in the health of education professionals should help Nursing students to build critical positions to conduct increasingly emerging discussions in the post-pandemic scenario.

Keywords: Professional exhaustion; social distance; COVID-19.

\section{INTRODUÇÃO}

A pandemia da COVID-19 provocou e tem provocado profundas transformações nos modos de vida nos contextos individuais e coletivos devido às suas implicações na saúde da população. O número crescente de casos e fatalidades ocorridos pela infecção com o vírus no mundo provocou e tem provocado um significativo impacto socioeconômico, político e psicossocial de proporções ainda não vivenciadas neste século. (BANERJEE; RAI, 2020). A extensão e a profundidade desse impacto ainda estão sendo avaliadas, ressaltando o caráter provisório dessas reflexões, mas, em contrapartida, a necessidade que as mesmas possam ser apresentadas, problematizadas e validadas, permitindo avanços importantes no conhecimento gerado durante e após a pandemia. (SCORSOLINI-COMIN; ROSSATO; SANTOS, 2020).

O contexto da pandemia motivou a implementação de mudanças sociais, principalmente com o objetivo de frear o número de casos da doença, tanto em contexto global, como no contexto nacional e local. No Brasil, a principal medida adotada com o objetivo de diminuir a ocorrência de novos casos da doença foi o distanciamento social a partir de março de 2020. Essa estratégia, quando seguida de forma sistemática e organizada pela população, pode ser particularmente útil, pois consiste em um procedimento cujo objetivo é diminuir as interações entre pessoas em uma comunidade na qual os indivíduos podem estar infectados, mas ainda não foram identificados. (WILDER-SMITH; FREEDMAN, 2020). Como as doenças transmitidas por gotículas respiratórias exigem certa proximidade entre as pessoas, como é o caso da COVID-19, o isolamento e o distanciamento social acabam sendo estratégias potencialmente eficazes na redução da transmissão. (WILDER-SMITH; FREEDMAN, 2020). Tais estratégias também têm sido investigadas considerando os contextos dos diferentes países, de modo a compreender que uma mesma ação pode ser implementada de maneiras distintas e que também pode promover resultados diferentes. (SCHWARTZ, 2020).

O contexto de distanciamento social adotado fez com que todas as atividades não essenciais que oferecessem risco de contágio entre pessoas devido à aglomeração fossem suspensas, quadro que perdurou no ensino superior brasileiro durante quase todo o ano de 2020. A maioria dos países adotou medidas para reduzir o contágio, de modo que mais de mais de um terço da população global esteve em isolamento ao longo de seis meses ou mais, em 2020, levando ao fechamento de escolas, universidades, instituições públicas, entre outros espaços. (MAIA; DIAS, 2020). 
Entre as atividades suspensas em 2020 estão as de ensino presencial que, apesar de serem de extrema relevância para os sujeitos, foram canceladas como medida de prevenção. Desse modo, o ensino presencial foi interrompido em seus mais diversos níveis de ensino devido à necessidade de evitar qualquer tipo de aglomeração, em uma tentativa de reduzir o contágio pelo vírus. (MARQUES, 2020). Embora movimentos de retomada de atividades presenciais em estados como Amazonas, Rio de Janeiro e São Paulo tenham ocorrido a partir de setembro de 2020, momento em que este estudo foi originalmente produzido, a maioria das instituições de ensino seguiu com atividades presenciais suspensas e com ações ofertadas de modo remoto. Modificações nesse cenário começaram a ocorrer de modo mais sistêmico a partir do final do primeiro semestre de 2021, com a retomada presencial e gradual das atividades de ensino nas diferentes esferas e mesmo com a oferta do ensino híbrido.

Milhares de estudantes e professores foram e têm sido impactados com as medidas de distanciamento adotadas pelos organismos de vigilância em saúde. A abrupta interrupção das atividades previamente planejadas em aulas presenciais, as quais associavam atividades teóricas e práticas, gera impactos a curto, médio e longo prazos. Especialmente para as populações socioeconomicamente vulneráveis, essa medida pode acentuar as desigualdades sociais no acesso a bens e serviços essenciais, como é a educação. (COSTA et al., 2020). Assim, destaca-se que os efeitos da interrupção das atividades presenciais de ensino podem afetar de modo diferente essas populações, de modo que não devemos nos apartar de uma discussão que também considere as assimetrias e as desigualdades que atravessam e constituem a educação no Brasil.

Com a suspensão das atividades de ensino presencial, outros recursos começaram a ser pensados como uma alternativa ou complementar diante do cenário que se instaurou com o distanciamento social. Em alguns contextos específicos, as práticas de ensino foram readequadas para outras modalidades, principalmente por meio de aulas via plataformas online, sendo que a adoção dessa modalidade de ensino para muitos estudantes e professores consistiu em algo novo, nunca antes vivenciado. (OLIVEIRA; LISBÔA; SANTIAGO, 2020).

Especificamente no ensino superior, o trabalho docente em tempos de pandemia tem se mostrado um desafio diante do contexto instaurado momentaneamente e da necessidade de adaptação devido à nova realidade imposta. Além dos ajustes inerentes à nova rotina no contexto da vida privada, os docentes tiveram que se adaptar a um novo cenário do mundo do trabalho sem que, em muitas situações, tivessem recursos disponibilizados pelas instituições de ensino ou competências específicas para as adaptações necessárias e essenciais para a condução das chamadas atividades remotas. (RIBEIRO; SCORSOLINI-COMIN; DALRI, 2020).

As novas atribuições estabelecidas aos docentes, somadas a outras exigências que já tinham em seus contextos de trabalho, têm exigido uma significativa capacidade de adaptação. O trabalho docente, em seus moldes tradicionais, já exige constantes atualizações, além de responsabilidade perante a sociedade, sendo que o empenho dos docentes contribui para o processo educacional exigindo resultados positivos. 
(PEREIRA; AMARAL; SCORSOLINI-COMIN, 2011) e no contexto de pandemia tem exigido ainda mais esforços por parte desses profissionais. Tais exigências estão associadas à sobrecarga devido ao acúmulo de funções, à necessidade de desenvolvimento de novos materiais de ensino, necessidade de desenvolvimento de novas interações mediadas pela tecnologia, de aquisição de conhecimentos técnicos para o manejo dessa tecnologia, além dos diversos desafios relacionados ao home-office, como adaptação das rotinas familiares e flexibilização de tempos e espaços para que as atividades profissionais possam ocorrer concomitantemente com as demandas domésticas que envolvem, em muitos casos, a concomitância com os desafios da parentalidade. Em um cenário de feminilização dessa profissão, os desafios podem ser ainda mais expressivos para que haja essa adaptação, sendo necessário adicionar a essa discussão uma perspectiva de gênero que considere que os efeitos da pandemia podem ser diferentes para professores e professoras. (SILVA et al., 2020; OLIVEIRA, 2020).

No contexto de ensino de Enfermagem, as práticas educativas via plataformas digitais, embora sejam bastante descritas na literatura em saúde (SANTOS et al., 2020), ainda representam um desafio, uma vez que as atividades desenvolvidas pelos docentes, principalmente em universidades, não se limitam ao ensino em sala de aula propriamente dito, mas também em ações de pesquisa, extensão, funções administrativas e estágios que, em muitos casos, representam o oferecimento de serviços para a sociedade. Desse modo, ao docente exige-se não somente a adaptação a um aspecto, mas a vários que são igualmente importantes e que não podem deixar de ser executados. (SCORSOLINI-COMIN; MELO; ROSSATO; GAIA, 2020).

Ao mesmo tempo, nesse contexto de pandemia a Enfermagem consiste em uma das principais profissões do que convencionou-se denominar linha de frente ao combate à doença, sendo que muitos docentes atuam profissionalmente em ambientes de saúde ou acompanham orientandos e estagiários que estão inseridos em contexto de atendimento em saúde. A partir desse panorama, observa-se que a atuação na docência em Enfermagem possui algumas especificidades, de modo que esses docentes, em sua maioria do sexo feminino, precisam lidar com extensas jornadas de trabalho, coordenação de atividades de ensino remoto com possíveis inserções na prática e, em muitos casos, com a própria prestação de serviço em equipamentos de saúde. (COSTA et al., 2020).

Diante desse cenário o docente se vê diante de inúmeros desafios que podem comprometer a sua saúde emocional. Reinventar-se em um contexto de dúvidas, medos e necessidades impostas pela realidade social é, no mínimo, complexo, sendo necessário a curto e longo prazo analisar os efeitos colaterais dessas adaptações. (COSTA et al., 2020). A própria necessidade de adaptação sugerida a esses profissionais como algo essencial para a manutenção de cargos e funções acaba exigindo tanto reflexões como processos de flexibilização e de aquisição de novos conhecimentos bastante centralizados em aspectos individuais, sobrepondo-se a esforços coletivos e que poderiam, de modo compartilhado, promoverem maior bemestar e acolhimento nesse período de maior mobilização. Em um contexto de trabalho 
coletivo que, por vezes, acaba sendo analisado por meio de adaptações e itinerários pessoais e individuais, desconsiderando as determinações sociais e laborais, emergem, de modo inequívoco, questionamentos sobre os processos de adoecimento decorrentes desse cenário. Nesse sentido, refletir sobre o estresse ocupacional de docentes em tempos de pandemia mostra-se pertinente principalmente analisando uma das formas mais recorrentes deste que é a síndrome de burnout.

A síndrome de burnout surge como consequência do estresse ocupacional, prolongado e crônico, que afeta as pessoas que desenvolvem seu trabalho em contato com os indivíduos, sendo bastante característico nos trabalhos da área da saúde, educação, justiça e serviço social. Leva os profissionais a sentimentos negativos, como tristeza, apatia, desmotivação e a exaustão emocional. (MASLACH; LEITER, 2016). Essa síndrome caracteriza-se pelo esgotamento emocional ou o sentimento de tensão emocional e esgotamento físico de um indivíduo relacionado ao seu trabalho, experimentando exaustão emocional, apatia e indiferença em relação ao trabalho, sentimentos de incompetência e diminuição da produtividade, entre outros aspectos que, na medida que se intensificam levam o sujeito a ter atitudes negativas em relação ao trabalho e ao ambiente que está inserido (BRIDGEMAN; BRIDGEMAN; BARONE, 2018), podendo levar ao adoecimento psíquico.

$\mathrm{Na}$ literatura científica, os relatos acerca de docentes acometidos pelo burnout são cada vez mais recorrentes (CARVALHO; MACÊDO, 2020; CARVALHO; MELO, 2020; SILVA et al., 2020), o que nos legitima a refletir, em um cenário de pandemia, como esses profissionais estariam submetidos a fatores desencadeadores desse tipo de adoecimento. Nessa perspectiva, este relato se propõe a apresentar a experiência de uma discussão virtual acerca do assunto com estudantes de Enfermagem de uma instituição privada. Este relato mostra-se relevante não apenas por permitir o debate sobre um importante tema no cenário da saúde ocupacional em Enfermagem, mas também como reflexão orientada para a prevenção em saúde mental durante o teletrabalho docente na pandemia da COVID-19. De igual monta, espera-se que tais apontamentos possam ser revisitados não apenas durante o curso da pandemia, mas também em um cenário posterior, promovendo debates sobre a saúde ocupacional e a atuação docente no ensino superior.

\section{DESENVOLVIMENTO}

\subsection{MÉTODO}

Trata-se de um relato de experiência descritivo e reflexivo, vivenciado durante o teletrabalho docente na pandemia de COVID-19. A experiência ocorreu durante a condução das atividades de uma disciplina ministrada virtualmente por uma plataforma disponibilizada em um Centro Universitário Privado de Ensino Superior do norte do Estado do Paraná.

A carga horária total da disciplina era de 51 horas, sendo dez horas destinadas à abordagem do tema doença do trabalho e qualidade de vida no trabalho. Os objetivos 
de tal disciplina eram conhecer, discutir e refletir sobre princípios de prevenção, proteção e promoção da saúde dos trabalhadores, bem como construir noções teóricas e práticas da enfermagem do trabalho, promovendo estratégias de promoção de saúde e qualidade de vida do trabalhador aos discentes matriculados na disciplina de Saúde ocupacional de uma Universidade Privada de Ensino Superior.

O período de experiência ocorreu de março a agosto de 2020, durante as aulas virtuais com três horas semanais, executadas em uma plataforma online da instituição, em que os discentes têm acesso aos materiais, links, videoaulas e atividades para desenvolver durante a pandemia. A elaboração deste estudo ocorreu a partir das atividades entregues em um fórum acadêmico e no chat organizado pela disciplina. As respostas obtidas nesses espaços interativos foram organizadas a partir dos temas emergentes, que foram submetidos à interpretação e à reflexão a partir da literatura da área, articulando tanto estudos produzidos antes da pandemia como produzidos no curso pandêmico, o que se apresenta como um desafio analítico significativo e mostra o caráter ainda impermanente das discussões aqui empreendidas. Acerca da produção científica sobre a pandemia da COVID-19, uma revisão narrativa foi conduzida para orientar a discussão dos dados trazidos a partir da experiência de ensino aqui problematizada.

\subsection{RESULTADOS E DISCUSSÃO}

Ao disponibilizar os materiais e a atividade no fórum acadêmico sobre o burnout e o teletrabalho, os estudantes manifestaram grande interesse pela temática. Diante disso, a docente compartilhou na plataforma online o conteúdo com a temática síndrome de burnout, esclarecendo que essa síndrome representa a descrição de exaustão ou extenuação e é específica do meio laboral. É caracterizada por respostas aos estressores interpessoais crônicos, ou seja, uma resposta prolongada aos períodos de estresse ocupacional. (MEDANHA; BERNARDES; SHIOZAWA, 2018).

A docente explicou que os professores universitários estão entre os profissionais com alto risco para desenvolver a síndrome de burnout, haja vista que esta é uma profissão que se relaciona diretamente com o ser humano e que, ao longo do tempo, desgasta-se pela baixa remuneração, por falta de reconhecimento, pela desmotivação, pelas relações interpessoais conflituosas, pela violência, dentre outros fatores também associados ao fazer docente. (CARLOTTO, 2002). Concomitante a isso, esclareceu aos discentes as implicações da atuação do enfermeiro do trabalho sobre a síndrome de burnout durante a pandemia da COVID-19 e a importância do apoio organizacional. Foi aberta uma discussão e, nesse momento, resgatou-se argumentos dos materiais que já haviam sido disponibilizados aos alunos nas aulas presenciais, motivando reflexões para o pensamento crítico para que, enquanto futuros profissionais de saúde ocupacional, auxiliem no bom desempenho de suas funções laborais.

Ações educativas e de conscientização para a prevenção e a manutenção da saúde e segurança são de grande relevância na formação de enfermeiros. Essas ações auxiliam os profissionais na construção de uma cultura de prevenção correspondente 
à responsabilidade social de cada instituição, possibilitando reduzir os índices de acidentes de trabalho, doenças profissionais e do trabalho, além de possibilitar cumprir a legislação trabalhista e civil no que se refere à saúde do trabalhador e, nesse caso, minimizar o estresse laboral (RIBEIRO; HIRAI; TESTON, 2018), em especial, a síndrome de burnout. Lançada essa explicação, percebeu-se uma linha de raciocínio e compreensão do estudante de saúde ocupacional.

Os discentes começaram a responder de forma solutiva e estratégica ao cuidado de saúde do trabalhador, esclarecendo que os docentes têm sido mais "cobrados" nesse período. Exemplificam esse evento narrando que, em uma sala de aula presencial, se todos os alunos têm a mesma dúvida, um deles levanta a mão e pergunta. Dessa forma, todos são respondidos e esclarecidos sobre a dúvida. Já em uma aula remota, como no contexto da educação a distância (EAD), se todos têm a mesma dúvida, os mesmos terão que perguntar, seja por meio de aplicativos de comunicação como o WhatsApp ou pela plataforma de aprendizagem ou via e-mail, gerando sobrecarga de trabalho e estresse no professor que precisa responder, em determinadas situações, várias vezes ao mesmo questionamento.

Os discentes mencionaram que durante as aulas presenciais o professor ministra aula no dia da disciplina e no teletrabalho, como a plataforma fica seis dias aberta a interações, no caso da instituição em tela, enquanto os estudantes estiverem acessando os conteúdos o professor precisará responder às dúvidas que surgirem no horário em que o aluno reservar para a atividade, causando uma sobrecarga de trabalho. Dessa maneira, durante a discussão virtual, os discentes sugeriram utilizar certas estratégias para que as cobranças, a sobrecarga e o excesso no trabalho não façam com que os docentes desenvolvam a síndrome de burnout e também outros tipos de adoecimento provocados no contexto laboral. Mencionaram que os docentes deveriam estipular metas, separar o momento de trabalho, descanso, hora de atividade, atividade física, reservar um tempo para leituras e demais atividades lúdicas (como ouvir música, dançar, conversar com os amigos pelas redes sociais) para não sofrer durante a pandemia e responder aos alunos somente em alguns momentos do dia ou apenas no horário que estariam em aula presencial.

Em relação a essas estratégias, há que se considerar que a experiência aqui narrada foi realizada no início da deflagração da pandemia e a partir das primeiras repercussões das ações de distanciamento e de isolamento social. Assim, tratava-se, à época, de um período de maior mobilização devido à necessidade de adaptação emergencial a um novo contexto de oferta de disciplinas e conteúdos. Nesse sentido, tanto alunos quanto professores passaram a ser submetidos a convites desenvolvimentais importantes em termos de aquisição de competências importantes para o período.

A sobrecarga do trabalho de docentes do ensino superior tem sido relatada na literatura científica e apresentada como uma problemática importante de ser considerada, principalmente pelo fato de provocar o adoecimento psíquico dos professores. Em instituições de ensino superior que já ofertavam a modalidade de cursos a distância, por exemplo, evidenciou-se que essa prática representava uma 
sobrecarga nas atividades desenvolvidas pelos docentes, inclusive em termos dos atendimentos aos estudantes. (ALONSO, 2010).

Em 2020, a transição do ensino presencial para o ensino remoto pode ser problematizada, ainda que em uma condição transitória, pois é um fenômeno que incidiu diretamente nos modos de ensino tradicionalmente adotados pelos professores, requerendo mudanças rápidas para as quais não estavam preparados emocionalmente e financeiramente. Tais necessidades - a de adaptação e de uma rápida resposta a alunos e instituições - tornaram-se elementos de sobrecarga emocional. As pesquisas acerca dessas adaptações e seus impactos ainda estão em curso. É importante ressaltar que, em casa, muitas vezes os computadores e equipamentos têm uma grande demanda por parte de pais, filhos e outros parentes que também precisam trabalhar/estudar no mesmo ambiente. Trabalhar nesse contexto pode ser uma tarefa potencialmente estressora, o que se aplica diretamente aos docentes de ensino superior. (SAHU, 2020).

Em uma população em que o estresse e a sobrecarga de trabalho já se fazem presentes, eventos como a pandemia da COVID-19 podem potencializar os sintomas psicopatológicos pré-existentes. O aumento do estresse também pode ser seguido por comportamentos relacionados à ansiedade, como distúrbios do sono e um estado geral de saúde percebido como mais baixo. Esse cenário pode ser potencializado pela necessidade de trabalho remoto aos docentes não como uma opção, mas como uma condição. (BESSER; LOTEM; ZEIGLER-HILL, 2020). Esses aspectos, quando associados, podem ser propulsores para o surgimento de sintomas da síndrome de burnout.

No grupo de discussão virtual, alguns discentes mencionaram que como "futuros enfermeiros do trabalho" acreditaram que em uma análise laboral teriam a percepção que os docentes estão "sufocados" com a pandemia, já que estes têm se esforçado para manter o ensino de qualidade e atender às particularidades de cada aluno, o que pode gerar maior vulnerabilidade para o burnout. Os discentes apontaram que, em especial, o enfermeiro do trabalho pode desenvolver ferramentas essenciais para preservar a saúde física e mental docentes em períodos de maior mobilização como o da pandemia, auxiliando na melhoria das relações interpessoais e do sujeito com o trabalho.

Entre as sugestões elencadas no fórum estão a organização de agendas de aula para alunos e professores, a diversificação de estratégias de ensino remoto, com atividades síncronas e assíncronas, construção de espaços específicos para a comunicação docente-discente, evitando duplicação de recursos e sobrecarga pelo excesso de informação, bem como a existência de um programa de acompanhamento em saúde do trabalhador com marcadores específicos sobre os regimes de trabalho existentes no contexto da pandemia. O acompanhamento emocional dos docentes com o subsídio das instituições de ensino superior também foi uma estratégia relatada, haja vista que muitos professores podem se sentir isolados diante desse contexto, ressaltando a importância de uma atenção coletiva e não focada individualmente. 0 cenário ocupacional nesse contexto de adaptação deve ser pensado coletivamente, processo que pode ser mediado pelos enfermeiros do trabalho. 
Os alunos também salientaram que as adaptações realizadas, sobretudo nos meses de maior mobilização, entre março e abril de 2020, devem ser acompanhadas longitudinalmente. Enfatizaram que a adaptação ao novo contexto não pode se sobrepor às necessidades de saúde expressas pelos docentes. Como estão se formando para serem futuros profissionais de saúde, esse olhar voltado à atuação docente destaca a capacidade desses estudantes empregarem os conhecimentos técnicos da área em um cenário mais próximo deles, das ações de ensinoaprendizagem.

É importante salientar que o propósito central do exercício da saúde no trabalho é a prevenção primária dos acidentes do trabalho e das doenças relacionadas ao trabalho, sendo que essa área tem desempenhado papel decisivo no planejamento da prestação de serviços de saúde e de segurança nos locais de trabalho (LINO et al., 2012), compondo um conhecimento essencial na formação do profissional de Enfermagem. Dessa forma, torna-se mister enfatizar no processo de ensino em Enfermagem a importância do cuidado da saúde ocupacional dos trabalhadores do ensino.

O ensino remoto representou mudanças significativas para a maioria dos professores que, na ausência de comunicação presencial, precisaram responder às necessidades dos alunos e das instituições que optaram pela manutenção das atividades de ensino na modalidade a distância. Entre as alterações destacadas no fórum estão a preparação de videoaulas, de atividades avaliativas realizadas a distância, o controle de frequência via plataformas de aprendizagem, atendimentos aos alunos por meio de diferentes aplicativos de comunicação, entre outras adaptações que têm sido aperfeiçoadas no decorrer da pandemia. Frente a esse cenário, as inovações desenvolvidas também permitiram um reposicionamento dos estudantes, que passaram a se engajar de modo mais propositivo e menos passivo nos processos de ensino-aprendizagem. (SUN; TANG; ZUO, 2020).

Considerar um contexto altamente estressor e de vulnerabilidade como o representado pela pandemia deve ser um norteador na formação desse profissional. Ainda que muitos dimensionamentos não possam ser estabelecidos a contento em função da transitoriedade desse cenário e das mudanças que ainda podem emergir, há que se desenvolver uma postura crítica com o aluno nesse processo de ensino e aprendizagem acerca dos conteúdos de saúde ocupacional. A partir de um olhar crítico e reflexivo, ampliam-se as balizas para a adoção de uma perspectiva analítica que possa não apenas compreender melhor o cenário e o seu impacto na saúde ocupacional, mas também atuar no desenvolvimento de estratégias que ofereçam respostas seguras em um momento de instabilidade.

A literatura tem apontado que profissionais com sintomas de ansiedade apresentam maior risco para o burnout. As redes de apoio compostas por colegas de trabalho podem auxiliar contra a ansiedade e a desesperança, principalmente em mulheres e em indivíduos negros, embora não protejam contra o estresse diretamente. (ATTELL; BROWN; TREIBER, 2017). Sendo assim, incentivar o trabalhador a desenvolver habilidades de enfrentamento, a resolução de conflitos, baseadas na eficácia, autoconfiança e controle eficaz de tarefas e funções auxilia na prevenção dessa 
síndrome. (MASLACH; LEITER, 2016). Obviamente que essas estratégias devem ser revisitadas no contexto da pandemia, não podendo se dar de modo apartado de uma discussão mais sistêmica e que afeta todo o mercado de trabalho. Assim, as possíveis soluções para o enfrentamento da crise decorrente da pandemia, sobretudo no contexto da educação superior privada, não podem ser pensadas apenas como uma questão centralizada nos recursos individuais de cada docente, mas de modo estrutural, promovendo formas de enfrentamento coletivo que possam repercutir, posteriormente, em uma melhor qualidade de vida e em estratégias pessoais mais bem-sucedidas, também considerando os importantes atravessamentos das desigualdades sociais, econômicas e educacionais que podem se revelar proeminentes nesse cenário em tela.

Em termos individuais, há que se considerar que dificuldades interpessoais e de comunicação, bem como comportamento cognitivo, podem levar pessoas a desenvolver burnout. (GEUENS et al., 2015). Trabalhadores que participam das decisões do trabalho são menos suscetíveis a dificuldades em exercer sua autonomia profissional e de experienciar perda de controle no processo de trabalho. Conflitos de trabalho não resolvidos, caracterizados pela falta de apoio e confiança aumentam o risco de burnout. (MASLACH; LEITER, 2016). Ressalta-se que, na instituição em tela, os docentes têm participado das decisões tomadas no setor laboral em meio à pandemia, com a oferta de apoio por parte do supervisor de ensino, o que pode ser uma condição protetiva para o estresse e para o burnout.

No que tange especificamente à disciplina de saúde ocupacional, os discentes atingiram os objetivos propostos, elaborando estratégias para que o docente tenha uma carga horária compatível com as suas demandas e que lhe permita uma atuação com qualidade. Essas estratégias envolvem reflexões importantes sobre o fazer docente e adaptações fundamentais para a continuidade dos processos de ensino e aprendizagem de modo remoto. Os ajustes assinalados envolvem, de modo conjunto, ações que devem ser lideradas pelos docentes e outras pelas instituições de ensino superior. Os alunos também enfatizaram a necessidade de que os docentes avaliem constantemente as suas condições de trabalho, preservando a qualidade do ambiente, com cuidados relativos à iluminação, ergonomia, entre outros. Por fim, mencionaram os benefícios decorrentes de um acompanhamento profissional em relação à saúde mental, o que pode se dar pela realização de psicoterapia online ou mesmo a partir de um acompanhamento subsidiado pelas instituições de ensino. Atentar-se aos sinais de cansaço, estresse e esgotamento profissional mostrou-se uma orientação perene, ressaltando a necessidade de um diálogo aberto para o compartilhamento desse momento de especial mobilização emocional.

Nessa pandemia as instituições de ensino superior, em especial as privadas, podem promover maior pressão emocional tanto em alunos como em docentes. Assim, é importante que os docentes possam conhecer mais sobre essa doença e seus sintomas, a fim de procurarem ajuda se necessário. Também as instituições de ensino, mesmo com os grandes desafios enfrentados diante da pandemia, devem estar atentas aos seus colaboradores. 
Pensar o trabalho e a docência a partir desses marcadores ainda é uma tarefa desafiadora e para a qual os alunos se mostram engajados, ainda que não reúnam todos os elementos necessários para uma análise que, de fato, permita a construção de novas inteligibilidades. Assim, a recomendação para que os alunos permanentemente desenvolvam posicionamentos críticos na área de saúde ocupacional pode ser um norteador importante para que novas reflexões possam ser endereçadas e atualizadas em função das mudanças que ainda incidirão em função da pandemia. Reforçar o compromisso crítico na formação do profissional de Enfermagem, desse modo, é uma recomendação fortemente associada ao que, neste relato, abarcamos sob a égide de uma estratégia de promoção de saúde.

\section{CONSIDERAÇÕES FINAIS}

O presente relato oportunizou importantes reflexões. A experiência em tela possibilitou avanços no que diz respeito a proporcionar conhecimento aos discentes sobre a síndrome de burnout ocorridas no ambiente de trabalho dos docentes, o que, por sua vez, possibilita a adoção de medidas de enfrentamento desta realidade e poderá ainda ser o norteador para novas pesquisas sobre a temática. A experiência, avaliada de modo positivo por estudantes e docentes, permitiu importantes reflexões sobre adaptação, autonomia, flexibilidade e responsabilidade durante a pandemia. Desse modo, permitiu-se que um conhecimento obrigatório à formação do profissional de Enfermagem, com uma reflexão que superou as aquisições cognitivas acerca do que é o adoecimento no trabalho ou como identificar e tratar o burnout.

A proposição do debate virtual fomentou uma discussão que teve como protagonista a própria figura do professor universitário, possibilitando uma reflexão pautada em um profissional essencial à formação dos estudantes e dos novos profissionais de saúde. Considerar as necessidades dessa categoria diante da pandemia foi uma oportunidade para expandir esses conhecimentos também para outros profissionais diretamente afetados por esse contexto, como os enfermeiros.

Nota-se, portanto, que apesar de a discussão ter se circunscrito a uma categoria em específico, a de docentes universitários, tal organização da disciplina não tornou a reflexão apartada de seu contexto mais amplo e de como outras categorias profissionais também têm sido impactadas por esse momento. Espera-se que esse movimento possa ser cada vez mais fomentado no ensino superior, ampliando as inteligibilidades e possibilitando que os novos profissionais de saúde estejam permanentemente engajados em um fazer crítico. Incorporar os estudos produzidos durante a pandemia acerca desse tema é um movimento essencial para amparar tais reflexões, permitindo avanços, ainda que em um contexto de instabilidade e de uma produção de conhecimentos ainda em trânsito. 


\section{REFERÊNCIAS}

ALONSO, K. M. A expansão do ensino superior no Brasil e a EaD: dinâmicas e lugares. Educação e Sociedade, Campinas, v.31, n.113, p.1319-1335, 2010.

ATTELL, B. K; BROWN, K. K; TREIBER, L. A. Workplace bullying, perceived job stressors, and psychological distress: gender and race differences in the stress process. Social Science Research, v.65, p.210-221, 2017.

BANERJEE, D; RAI, M. Social isolation in Covid-19: the impact of loneliness. International Journal of Social Psychiatry, 2020.

BESSER, A.; LOTEM, S.; ZEIGLER-HILL, V. Psychological stress and vocal symptoms among university professors in Israel: implications of the shift to online synchronous teaching during the COVID-19 pandemic. Journal of Voice, 2020.

BRIDGEMAN, P. J.; BRIDGEMAN, M. B.; BARONE, J. Burnout syndrome among healthcare professionals. The Bulletin of the American Society of Hospital Pharmacists, v.75, n.3, p.147-152, 2018.

CARLOTTO, M. S. A síndrome de burnout e o trabalho docente. Psicologia em Estudo, Maringá, v.7, n.1, p.21-29, 2002.

CARVALHO, M. P.; MACÊDO, M. E. C. A síndrome de burnout em docentes. ID Online Revista de Psicologia, v.14, n.50, p.284-301, 2020.

CARVALHO, L. O. T. D.; MELO, R. L. Síndrome de burnout: fatores de risco aos docentes de enfermagem em uma instituição de ensino superior. Interfaces do Conhecimento, v.2, n.2, 2020.

COSTA, R. et al. Ensino de enfermagem em tempos de COVID-19: como se reinventar nesse contexto? Texto \& Contexto-Enfermagem, Florianópolis, v.29, 2020.

GEUENS, N. et al. Interpersonal behavior in relation to burnout. Nursing Management, v.22, n.8, 2015.

LINO, M. M., et al. Enfermagem do trabalho à luz da visão interdisciplinar. Saúde \& Transformação Social, Florianópolis, v.3, n.1, p.85-91, 2012.

MAIA, B. R.; DIAS, P. C. Ansiedade, depressão e estresse em estudantes universitários: o impacto da COVID-19. Estudos de Psicologia, Campinas, v.37, 2020.

MARQUES, R. A ressignificação da educação e o processo de ensino e aprendizagem no contexto de pandemia da COVID-19. Boletim de Conjuntura, v.3, n.7, p.31-47, 2020.

MASLACH, C; LEITER, M. P. Understanding the burnout experience: recent research an its implications for psychiatry. World Psychiatry, Milan, v.15, n.2, p.103-111, 2016.

MEDANHA, M. H; BERNARDES P. F; SHIOZAWA, P. Desvendando o burnout: uma análise interdisciplinar da síndrome do esgotamento profissional. São Paulo: LTR, 2018. 
OLIVEIRA, A. L. A espacialidade aberta e relacional do lar: a arte de conciliar maternidade, trabalho doméstico e remoto na pandemia de COVID-19. Revista Tamoios, v.16, n.1, p.154-66.

OLIVEIRA, M. A. M.; LISBÔA, E. S. S; SANTIAGO, N. B. Pandemia do Coronavírus e seus impactos na área educacional. Pedagogia em Ação, v.13, n.1, p.17-24, 2020.

PEREIRA, H. O. S.; AMARAL, M. T.; SCORSOLINI-COMIN, F. Avaliação de sintomas de estresse em professores universitários: qualidade de vida no fazer docente.

Educação, Rio Claro, v.21, n.37, p.1-20, 2011.

RIBEIRO, B. M. S. S.; HIRAI, V. H. G.; TESTON, E. F. A redução de perícias médicas baseado na gestão de absenteísmo, rotatividade e qualidade de vida no trabalho.

Revista Eletrônica Gestão \& Saúde, v.9, n.3, p.393-403, 2018.

RIBEIRO, B. M. S. S.; SCORSOLINI-COMIN, F.; DALRI, R. C. M. B. Ser docente en el contexto de la pandemia de COVID-19: reflexiones sobre la salud mental. Index de Enfermería, v.29, n.3, p.1-5, 2020.

SAHU, P. Closure of universities due to coronavirus disease 2019 (COVID-19): impact on education and mental health of students and academic staff. Cureus, v.12, n.4, p.e7541. 2020.

SANTOS, S. M. R. et al. Docência superior na formação EAD no curso de enfermagem: revisão bibliográfica. Revista Eletrônica Acervo Científico, v.8, e2204, 2020.

SCHWARTZ, F. P. Distanciamento social e o achatamento das curvas de mortalidade por COVID-19: uma comparação entre o Brasil e epicentros da pandemia. Thema, v.18, n.especial, p.54-69, 2020.

SCORSOLINI-COMIN, F.; MELO, L. P.; ROSSATO, L.; GAIA, R. S. P. Distance learning in nursing training: reflections on the COVID-19 pandemic. Revista Baiana de Enfermagem, v.34, e36929, 2020.

SILVA, C. R. et al. Terapia ocupacional na universidade pública e ações de enfrentamento à Covid-19: singularidades e/nas multiplicidades. Revista Brasileira de Terapia Ocupacional, v.4, n.3, p.351-70, 2020.

SILVA, L. C. N. et al. Síndrome de burnout entre docentes de um centro universitário do Acre. ID Online Revista de Psicologia, v.14, n.50, p.1271-1281, 2020.

SUN, L.; TANG, Y.; ZUO, W. Coronavirus pushes education online. Nature Materials, v.19, n.6, p.687-687, 2020.

WILDER-SMITH, A; FREEDMAN, D. O. Isolation, quarantine, social distancing and community containment: pivotal role for old-style public health measures in the novel coronavirus (2019-nCoV) outbreak. Journal of Travel Medicine, v.27, n.2, taaa020, 2020. 